\title{
Antioxidant dressing therapy versus standard wound care in chronic wounds (REOX study): Study protocol for a randomized controlled trial
}

Inés María Comino-Sanz ( $\square$ icomino@ujaen.es)

Universidad de Jaen https://orcid.org/0000-0002-6822-1892

María Dolores López-Franco

Universidad de Jaen

Begoña Castro

Histocell

Pedro Luis Pancorbo-Hidalgo

Universidad de Jaen

Study protocol

Keywords: Chronic wound, Wound healing, Hard-to-heal wounds, Antioxidant dressing, and Oxidative stress.

Posted Date: May 19th, 2020

DOI: https://doi.org/10.21203/rs.3.rs-16762/v2

License: (a) This work is licensed under a Creative Commons Attribution 4.0 International License. Read Full License

Version of Record: A version of this preprint was published at Trials on June 8th, 2020. See the published version at https://doi.org/10.1186/s13063-020-04445-5. 


\section{Abstract}

Background: A wound that does not heal in the orderly stages of the healing process or does not heal within three months is considered a chronic wound. Wound healing is impaired when the wound remains in the inflammatory stage for too long. A range of factors can delay the healing process: imbalance between proteases and protease inhibitors in the wound bed; bacterial colonization and the presence of biofilm; and oxidative stress.

Recently, wound management has improved significantly. A new antioxidant dressing has been developed, which combines an absorbent matrix obtained from Locust Bean Gum (LBG) galactomannan and a hydration solution with curcumin and $\mathrm{N}$-acetylcysteine (NAC). This dressing combines the advantages of moist healing in exudate management and free radical neutralization, achieving wound reactivation.

The primary aim of this study is to compare the effect of antioxidant dressing on chronic wound healing with the use of standard wound dressing in patients with hard-to-heal wounds.

Methods: We will conduct a multicentre, single blinded, randomized, controlled trial with parallel groups. Participants will be selected from three primary public health-care centres, located in Andalucía (southern Spain). Patients will be randomized into an intervention group (antioxidant dressing) or control group (standard wound dressing). Assessment will be carried out in weeks 2, 4, 6 and 8. Follow-up will be of 8 weeks or complete healing, if it happens earlier.

Discussion: The findings from this study should provide scientific evidence on the efficacy of the antioxidant dressing as an alternative for the treatment of chronic wounds. This study fills some of the gaps in the existing knowledge about patients with hard-to-heal wounds.

Trial registration: ClinicalTrials.gov, NCT03934671. Registered on 2 May 2019.

\section{Background}

A wound that does not heal in the orderly stages of the healing process or does not heal within three months is considered a chronic wound, also known as hard-to-heal wounds or non-healing wounds [1]. Hard-to-heal wounds have been defined as any wound that has not healed by $40-50 \%$ after four weeks of good standard of care [2]. Chronic wounds are a major clinical and economic problem for healthcare institutions because of their impact on the quality of life of patients and caregivers [3-5]. Wound healing is impaired when the wound remains in the inflammatory stage for too long [1]. A range of factors that can delay the healing process have been described: the imbalance between proteases and protease inhibitors in the wound bed [6-8]; bacterial colonization and presence of biofilm [9-11]; and oxidative stress $[1,4-5,12]$. 
Matrix metalloproteinases (MMPs) and their inhibitors contribute to the balance between extracellular matrix degradation and deposition, creating an equilibrium that is essential for timely and coordinated healing $[7,8]$. However, in chronic wounds, the inflammatory response is augmented, and this balance become disrupted. This situation is characterized by elevated levels of proinflammatory cytokines and MMPs. Therefore, wound dressings aimed at sequestering the MMPs within the wound milieu is one particular area of interest as an alternative wound management strategy [6].

Biofilm is associated with impaired epithelialization and granulation tissue formation, and promotes a low-grade inflammatory response that interferes with wound healing $[9,10]$. Over $90 \%$ of chronic wounds have an aggregation of microorganism-forming biofilm [13]. Biofilm is a survival mechanism that confers to bacteria and other microorganisms the ability to resist environmental stressors and antimicrobials due to a variety of reasons, including low metabolic activity [11].

An excess of reactive oxygen species (ROS), including singlet oxygen, hydrogen peroxide and hydroxyl radicals, causes a pro-inflammatory environment in the wound bed $[14,15,16]$, attracting more inflammatory cells into the wound and creating a negative feedback loop that could delay or prevent wound closure $[1,4,5,17,18]$. The strong oxidizing capabilities of ROS can therefore damage many of the cells of molecules and structures [12]. It has been proposed that the use of a dressing that can exert an antioxidant effect on wound exudates might restore the appropriate ROS balance [19].

Recently, wound management has improved significantly. New materials and bioactive wound dressings have been developed that promote a favourable environment and promote active wound healing treatment, such as collagen dressings [20,21]; MMPs modulators dressings [22]; and dressings with antioxidant activity of inhibitors of free radical reactions [23,24].

Among these new advanced products, there is an antioxidant dressing (Reoxcare $\AA$ ) that was developed by Histocell (Bizkaia, Spain). This dressing combines an absorbent matrix obtained from Locust Bean Gum (LBG) galactomannan with antioxidant properties [25] and a hydration solution with curcumin $[26,27]$ and $\mathrm{N}$-acetylcysteine (NAC) $[28,29]$. Curcumin is a natural phenol from the rhizome of the plant Curcuma longa that has been used for over 2000 years as an antioxidant, anti-inflammatory and specifically in wounds, to improve healing [30-32]. NAC is widely applied as an antioxidant molecule, and recently successfully for the treatment of wounds [28,33]. These three components act as free radicals scavengers, as two of them also have a synergistic antioxidant effect [34]. Due to the innovative design, this antioxidant dressing combines the advantages of moist healing in exudate management and free radical neutralization, achieving wound reactivation. In addition, preliminary observations suggest that this antioxidant dressing may have anti-biofilm activity, to eliminate and prevent re-formation [24]. These findings suggest that the dressing may represent a new advanced alternative for the management of hard-to-heal wounds.

This dressing with antioxidant properties has been tested in animal models and in case series of patients with acute wounds and chronic wounds of various aetiologies (venous ulcers, neuropathic, post-surgical), pressure ulcers and neuroischemic diabetic foot ulcers, showing favourable results in activating the 
healing process $[33,35]$. Data have also been published that reflect an estimate of the cost-benefit of treatment with antioxidant dressings in hard-to-heal wounds with venous vascular aetiology [36]. However, there is yet to be a study comparing this new antioxidant dressing with standard wound dressings that maintain a moist environment, used in routine clinical practice for the treatment of chronic wounds. This trial is aimed at filling this gap in knowledge.

\section{Hypotheses:}

The hypotheses for the trial are:

The use of the antioxidant dressing will reduce the percentage of non-viable tissue in the wound bed more than standard wound dressings.

The use of the antioxidant dressing will increase new granulation tissue formation with respect to standard wound dressings.

The use of the antioxidant dressing will produce a higher rate of wound healing than when using standard wound dressings.

\section{Study objectives:}

The primary aim of this study is to compare the effect of an antioxidant dressing on the healing of chronic wounds with the use of dressings that create a moist environment (standard clinical practice) in patients with hard-to-heal wounds.

The secondary aims are to measure the intra-patient variation over time in the percentage of non-viable and granulation tissue in the wound bed and to measure the reduction in the wound area. The variation in the percentage of wound area covered by non-viable tissue is important because its reduction is an early sign of the activation of the healing process (which is expected to be an effect of the new dressing).and assessment, according to the Standard Protocol Items: Recommendations for Interventional Trials (SPIRIT) guidelines (Additional File 1).

\section{Methods}

\section{Design}

The REOX study will conduct a multicentre, single blinded, randomized, controlled trial with parallel groups. Figure 1 presents an overview of the schedule for enrolment, intervention and assessment, according to the Standard Protocol Items: Recommendations for Interventional Trials (SPIRIT) guidelines (Additional File 1).

\section{Setting}


The REOX study will run in three primary health-care centres (one urban and two rural centres) of the Andalusian Health Service in Spain. The centres were selected based on having advanced-practice wound nurses working in them, who could participate in the study. (detailed in Additional File 2).

\section{Participants}

Patients are eligible according the inclusion and exclusion criteria presented in Table 1. The sample was recruited from patients with wounds treated in one of the healthcare centres engaged in the study.

Table 1. REOX trial inclusion and exclusion criteria

\begin{tabular}{|c|c|}
\hline \multicolumn{2}{|l|}{ REOX trial inclusion and exclusion criteria } \\
\hline Inclusion criteria & Exclusion criteria \\
\hline $\begin{array}{l}\text { - Patients over } 18 \text { years of age. } \\
\text { - Patients with leg ulcers (venous, ischemic, traumatic or diabetic foot ulcer). } \\
\text { - Patients with dehisced surgical wounds healing by second intention. } \\
\text { - Patients with pressure ulcers. } \\
\text { - Wound area between } 1 \text { and } 250 \mathrm{~cm}^{2} \text {. (Wounds larger than } 250 \mathrm{~cm}^{2} \text { are quite } \\
\text { infrequent and it was decided not to include because such a large wound could } \\
\text { be more difficult to heal) }\end{array}$ & $\begin{array}{l}\text {-Systemic inflammatory disease or } \\
\text { oncological disease. } \\
\text { - Wounds with clinical signs of } \\
\text { infection. } \\
\text { - Terminal situation (life } \\
\text { expectancy less than } 6 \text { months). } \\
\text { - Ulcers from other aetiologies: } \\
\text { tumours, infectious. } \\
\text { - Wounds treated with negative } \\
\text { pressure therapy. } \\
\text { - Pregnancy. } \\
\text { - History of sensitivity or allergy } \\
\text { to any of the components of the } \\
\text { study dressing. }\end{array}$ \\
\hline
\end{tabular}

- Appearance of allergies or hypersensitivity to the dressing.

- Death.

- Hospital admission that interrupts the treatment in the primary healthcare centre.

-Changing the patient's residence, if the new residence is at a different health centre. 


\section{Sampling}

Sample size was estimated based on 2 possible outcomes:

a) Detecting a mean difference of 2 points in the total score with the RESVECH 2.0 scale for chronic wound healing [37] between intervention group and control group with a standard deviation of 2.0 (5\% type 1 error and $20 \%$ type 2 error) based on data published by Castro et al. who found a reduction of 8 points in RESVECH 2.0 at 4-weeks of treatment (this is a $23 \%$ reduction)[24]. We used a 2 points reduction for sample estimation, as minimum clinically significant difference; in order to have the study powered enough to detect just this small difference. The sample size needed is 17 patients per group.

b) Time for achieving a $50 \%$ reduction of the ulcer area; estimating an average of 49.5 days in the control group and 30.0 days in the intervention group with a standard deviation of 20.0 days (5\% type 1 error and $20 \%$ type 2 error); based on the study by Lee [38]. The sample size needed is 18 patients per group.

An additional $30 \%$ is added to compensate possible lost patients in follow-up, so the final sample size is 54 patients ( 27 patients in each group). It is expected that each one of the three healthcare centres recruit 18 patients for the study.

\section{Randomization and blinding}

Patients ( $\mathrm{n}=54)$ will be randomized into an intervention group (antioxidant dressing) or control group (standard clinical practice using dressings that create a moist environment). The block randomization scheme was generated by using the software EPIDAT. Participants will be randomized to receive antioxidant dressing or standard wound dressing with a 1:1 allocation ratio (Figure 2) using stratified block randomization with a fixed block size per centre. Once the patient has accepted to participate and meets the inclusion criteria, the clinical nurse will open an opaque, sealed envelopes sequentially numbered containing a sheep (folded 4 times) with the group allocation. Neither the patient nor the clinical nurse is blinded to the treatment received; data and wound photographs will be codified, the assessor who evaluates the photographs and the data analyst will be blinded to the treatment.

\section{Intervention and control}

The REOX study has two arms (see Fig.2). Both arms included cleansing with saline solution and debridement to remove devitalized tissues, if needed, according to recommendations from clinical practice guidelines.

In the intervention group, the antioxidant dressing will be applied over the wound bed and covered with a secondary adhesive dressing. The dressing will be kept in place for 2 to 3 days, according to the recommendations of the manufacturer.

In the control group (standard practice), a dressing that creates a moist environment will be applied over the wound bed (such as hydrocolloid, alginate, polyurethane foam or silver dressing, according to the 
nurse's judgement and the availability of the products at the centre) and covered with a secondary adhesive dressing if necessary. The dressing will be kept in place for 2 to 3 days, according to the recommendations of the manufacturer.

To improve patient adherence to the treatment (both groups), the clinical nurse treating the patient will reinforce at each dressing change how important it is to keep the dressing in place until the next visit, and explain that if the patient is experiencing pain, itching or other wound related symptom, he/she should consult the nurse, but not remove the dressing. Any dressing related symptom will be recorded as a possible adverse effect.

\section{Data collection and management}

Each patient included in the study will be assessed by one clinical nurse at baseline and every 2 weeks (week 2, 4, 6 and 8). Figure 1 shows the variables collected at each assessment. The follow-up of patients in both groups will be prolonged until week 8 or complete healing of the wound, if it occurs earlier. A follow up of 8 weeks was considered as adequate for this study, because the main action of the antioxidant dressing tested is to reactivate the healing process by controlling the excess of ROS; according to the previous study by Castro et al. [35] at 4 weeks an significant number of wounds had improved. In order to promote participant retention and complete follow-up, the nurse will conduct the monitoring of patients in the healthcare centre. Furthermore, the principal investigator will have the primary responsibility for study monitoring; a formal data monitoring committee is not deemed necessary because there is not access to protected information and there is a limited amount of personal information.

To monitor wound healing, several variables will be used:

- Wound area, direct measurement of length and width with a graduated ruler (Kundin method) [39].

- Wound area estimated using a digital photography using the software Helcos $[40,41]$. This software is an integrated wound management system, which allows for estimation of the wound area and the relative percentage of tissue types in the wound bed (granulation, slough and necrotic tissue).

- Score in the RESVECH 2.0 scale for chronic wound healing [37].This scale assesses 6 aspects: wound size; depth/affected tissues; wound edges; type of tissue in the wound bed; exudate; and infection/inflammation. More score points means improvement in the healing process, so the score ranges from 0 (the worst possible status of the wound) to 35 point (wound healed).

Other variables to be recorded are demographic characteristics, anthropometric characteristics, pain level, concomitant medical diagnosis and clinical antecedents, nutritional status, consumption of drugs, tobacco habit, ankle-brachial index (ABI) and treatment with compressive therapy. No biological specimen will be collected or stored in this study.

\section{Outcomes}


The primary outcome is the improvement in wound healing, with two endpoints: a) increase in the score of the RESVECH 2.0 scale [37] (score at baseline minus score at 8 weeks); and b) wound area reduction (area at baseline minus area at 8 weeks). Although the RESVECH 2.0 score includes the assessment of the wound area, it was decided to use the reduction in wound area as a specific endpoint in order to have a direct measurement of wound size (in spite of not making the sample size estimation with this outcome because there were no data available from previous studies). Additionally, the values at 2, 4 and 6 weeks for each endpoint will be also calculated. These endpoints are chosen for the clinical relevance of the antioxidant dressing because this product is well-known for removing the non-viable tissue (necrotic or devitalized) in the wound bed, for promoting the production of granulation tissue and for activation of the healing process $[35,42]$.

Secondary outcomes are: time to achieve a $50 \%$ reduction in wound area; time elapsed until removal of non-viable tissue from wound bed; reduction of wound area; number of completely healed wounds; pain level; and the area of wound with bacterial load (clinical signs of infection or measurement of surfaces with bacteria).

\section{Data analysis plan}

A researcher blinded to group allocation will do data analysis. All analyses will be performed with SPPS Statistics software (IBM Corp.; Armonk, NY). Data will be expressed as frequency and percentages for categorical variables and mean, standard deviation (SD), range and median for quantitative variables depending on the distribution (normality will be checked with the Shapiro-Wilk test). The statistical significance level will be set at $p<0.05$.

For the primary outcome, improvement in wound healing, the wound area reduction at 8 weeks in the intervention group compared with the standard care group will be tested by a difference of means test. The increase in RESVECH 2.0 [37] score at 8 weeks between both groups will be examined by a difference of means test ( $t$ test for independent groups or Mann-Whitney test, depending on normal distribution). The effect size will be estimated by Cohen's d statistic. Additionally, a repeated-measures analysis (ANOVA or Friedman test) and survival analysis (Kaplan-Meier) will be used for analysing the evolution over time.

For secondary outcomes, proportions and categorical variables will be tested using a chi-squared test, and continuous variables will be tested using a mean difference test (parametric or non-parametric).

Analyses will be performed over groups as randomized (intention-to-treat analysis). Missing data of the outcomes will be tested if are missing at random; otherwise the last observation carried forward approach will be used for imputation [43]. Also, sensitivity analyses over all the complete cases (per protocol analysis) will be performed to check for inconsistencies.

A subgroup analysis by wound type will be performed to explore whether the effect of the intervention differs in any type of wound according to its aetiology. Moreover, another subgroup analysis by primary 
care centre to check if there are any difference in outcomes according to the centre.

No interim analyses or formal stop study rules are planned; no detrimental problems for patients are anticipated because this new dressing has been previously tested on a series of patients with wounds of various aetiologies without any serious adverse effect [35].

\section{Oversight and monitoring}

The project team at the University of Jaén is responsible for the design, preparation, and coordination of the trial, including dissemination of the results. This team meets every month to discuss progress and solve arising problems. A steering committee, formed by two professors of the Nursing department of the University of Jaén and a member of Histocell, will supervise the trial. This committee will meet twice a year during the development of the study and at the completion of data collection. For each participating primary care health centre will be a clinical nurse who will be responsible for recruiting patients, obtaining informed consent and coordinating the trial at the centre. Additionally, some site visits are planned every 3-4 months to audit the trial and monitor compliance with the study design. Periodical reports with data on recruitment and follow-up rates will be sent to the principal researcher every month. Any deviation from the protocol will be fully documented by a breach report. Any significant amendments to the protocol will be communicated to the relevant parties and updated in the trial register.

\section{Dissemination plan}

The findings obtained in this study will be disseminated at conferences on wound management (mainly at European level) and at other relevant national and international meetings. The key findings could be early communicated by social media and the website of the research group; and will be updated in the trial register. The full report of the study will be submitted for publication in a wound journal, preferably an open access journal.

\section{Discussion}

This randomized clinical trial will be the first to compare the effect on chronic wound healing of antioxidant dressing with the use of dressing that creates a moist environment (standard care) in patients with hard-to-heal wounds.

Currently, through the research of scientists, engineers and manufacturers, we are witnessing an increase in scientific knowledge about wound healing; this is associated with the development of products that promote positive results and mitigate negative factors in wounds. All of these research findings and resources are transferred to clinical practice through evidence-based wound management [44].

The dressing with antioxidant properties has been previously tested in animal models [33] and in patients with acute and chronic wounds of various aetiologies, showing potential to activate the healing 
process [35]. The antioxidant effect of NAC may play a role for tissue repair under normal and pathological conditions, improving the wound healing $[28,42]$.

In addition, some recently published data point to a positive cost-benefit balance for the treatment of venous ulcers with antioxidant dressing $[24,36]$.

A potential limitation of this study may arise for the inclusion of wounds with different aetiologies, so any imbalance in allocation could confound the findings. This is important for diabetic foot ulcers and for venous ulcers. This limitation will be managed during the study by trying to balance the type of wound included and by sub-groups analysis (after data collection).

However, thus, far no study has been conducted comparing this new antioxidant dressing with standard dressings to maintain a moist environment. If our results confirm the hypothesis, this randomized clinical trial could help to provide new clinical evidence of the efficacy of the antioxidant dressing as an alternative for the treatment of chronic wounds. This study aims to fill some of the gaps in the existing knowledge of advanced therapies for chronic wounds.

\section{Trial Status}

The study is registered on ClinicalTrials.gov (NCT03934671). Registered on 2 May 2019

(https://bit.ly/3etFnrD). The Committee of ethics of Jaén approved the study in April 2019, reference number 0645-N-19. This Protocol Version is Version 2.0; April 2020. Recruitment began in September 2019. The expected date for recruitment completion is December 2020.

\section{Abbreviations}

ABI: Ankle-brachial index

NAC: N-acetylcysteine

RESVECH 2.0: Scale for monitoring the healing of the wound

ROS: Reactive oxygen species

\section{Declarations}

\section{ACKNOWLEDGEMENTS}

The authors would like to acknowledge the University of Jaén and Histocell for providing support and training, and the professionals and patients who participate in the study.

\section{FUNDING}


The research was funded by the research group Nurse and innovation in healthcare, through the 20192020 Plan for Research Support of the University of Jaén. ICS has a scholarship of the Ministry of Innovation, Science and Universities of Spain. The funder played no part in study design; collection, management, analysis, and interpretation of data; writing of the report; and the decision to submit the report for publication.

\section{AVAILABILITY OF DATA AND MATERIALS}

The datasets generated and/or analysed during this study are not publicly available due to individual privacy considerations, but can be available from the corresponding author upon reasonable request.

\section{AUTHORS' CONTRIBUTIONS}

ICS and PPH developed the initial study protocol. BC and MLF supported by reading the manuscript critically and providing relevant comments. All authors reviewed, read and approved the final manuscript. All named authors adhere to the authorship guidelines of Trials, authors have agreed to publication and have contributed to writing the manuscript, no professional writer have been involved.

\section{ETHICS APPROVAL AND CONSENT TO PARTICIPATE}

This study was approved by the Research Ethics Committee of Jaén with the number 0645-N-19. It was approval by the Spanish Agency for Medicines and Medical Devices. The trial's protocol was registered at ClinicalTrials.gov on 2 May 2019 (NCT03934671). Study participants will provide written consent prior to participation; all participants will sign informed consent before enrolment in the study. These materials are available from the corresponding author on request. Any changes to the protocol or severe adverse events will be reported to Committee. Confidentiality of the data and the results of monitoring will be protected.

\section{CONSENT FOR PUBLICATION}

Not applicable.

\section{COMPETING INTERESTS}

$\mathrm{BC}$ is an employee of Histocell (collaborating company). The other authors declare that they have no competing interests.

\section{References}

1. Guo S, Di Pietro LA. Factors afecting wound healing. J Dent Res. 2010; 89(3):219-229.

2. Atkin L, Bućko Z, Conde Montero E, Cutting K, Moffatt C, Probst A, Romanelli M, Schultz GS, Tettelbach W. Implementing TIMERS: the race against hard-to-heal wounds. J Wound Care 2019; 
28(3 Suppl 3):S1-S49.

3. Diegelmann RF, Evans MC. Wound healing: an overview of acute, fibrotic and delayed healing. Front Biosci. 2004;9:283-9.

4. Drew P, Posnett J,Rusling L. The cost of wound care for a local population in England. Int Wound J. 2007;4(2):149-55.

5. Hopman WM, Buchanan M, Vandenkerkhof EG, Harrison MB. Pain and health-related quality of life in people with chronic leg ulcers. Chronic Dis Inj Can. 2013;33(3):167-74.

6. McCarty SM, Percival SL. Proteases and Delayed Wound Healing. Adv Wound Care. 2013;2(8):438447.

7. Schultz GS, Wysocki A. Interactions between extracellular matrix and growth factors in wound healing. Wound Repair Regen. 2009;17(2):153-62.

8. Sabino F, Ulrich auf dK. Matrix metalloproteinases in impaired wound healing. Metalloproteinases In Medicine. 2015;2:1-8.

9. James GA, Swogger E, Wolcott R et al. Biofilms in chronic wounds. Wound Repair Regen 2008; 16(1):37-44.

10. Metcalf DG, Bowler PG. Biofilm delays wound healing: A review of the evidence. Burns Trauma. 2013;1(1):5-12.

11. Mendoza RA, Hsieh J, Galiano RD. The Impact of Biofilm Formation on Wound Healing. Wound Healing - Current Perspectives. 2019. doi: 10.5772/intechopen.85020

12. Schaffer SW, Jong CJ, Mozaffari M. Role of oxidative stress in diabetes-mediated vascular dysfunction: unifying hypothesis of diabetes revisited. Vascul Pharmacol.2012;57(5-6):139-49.

13. Attinger C, Wolcott R. Clinically addressing biofilm in chronic wounds. Wound Care. 2012; 1(3): 127132.

14. Menke NB, Ward KR, Witten TM et al. Impaired wound healing. Clin Dermatol. 2007; 25:19-25.

15. Vermeij WP, Backendorf C. Skin cornification proteins provide global link between ROS detoxification and cell migration during wound healing. PLoS ONE. 2010; 5(8):e11957.

16. Wagener F, Carels $C$, Lundvig D. Targeting the redox balance in inflammatory skin conditions. Int $J$ Mol Sci. 2013; 14(5):9126-9167.

17. Roy S, Khanna S, Nallu K et al. Dermal wound healing is subject to redox control. Mol Ther. 2006; 13(1):211-220.

18. Fitzmaurice SD, Sivamani RK, Isseroff RR. Antioxidant therapies for wound healing: a clinical guide to currently commercially available products. Skin Pharmacol Physiol. 2011; 24(3):113-126.

19. Sen CK. The general case for redox control of wound repair. Wound Repair Regen. 2003; 11(6):431438.

20. Singh O, Gupta SS, Soni M, Moses S, Shukla S, Mathur RK. Collagen dressing versus conventional dressings in burn and chronic wounds: a retrospective study. J Cutan Aesthet Surg. 2011;4(1):12-6. 
21. Shekhter AB, Fayzullin AL, Vukolova MN, Rudenko TG, Osipycheva VD, Litvitsky PF. Medical Applications of Collagen and Collagen-Based Materials. Curr Med Chem. 2019;26(3):506-516.

22. Krejner A, Grzela T. Modulation of matrix metalloproteinases MMP-2 and MMP-9 activity by hydrofiber-foam hybrid dressing - relevant support in the treatment of chronic wounds. Cent Eur $\mathrm{J}$ Immunol. 2015;40(3):391-394.

23. Klebanov GI, Liubitskiĭ OB, Il'ina SE, Medusheva EO, Ryl'tsev VV, Filatov VN. Antioxidant activity of inhibitors of free radical reactions in the dressing materials for wound healing. Biomed Khim. 2006; 1:69-82.

24. Castro B, Palomares T, Azcoitia I et al. Development and preclinical evaluation of a new galactomannan-based dressing with antioxidant properties for wound healing. Histol Histopathol. 2015; 30(12):1499-1512.

25. Petkova N, Petrova I, Ivanov I, Mihov R, Hadjikinova R, Ognyanov M, Nikolova V. Nutritional and antioxidant potential of carob (Ceratonia siliqua) flour and evaluation of functional properties of its polysaccharide fraction. J. Pharm. Sci. \& Res. 2017;9(11):2189-2195.

26. Hu P, Huang P, Chen MW. Curcumin reduces Streptococcus mutans biofilm formation by inhibiting sortase A activity. Arch Oral Biol. 2013;58(10):1343-1348.

27. Packiavathy IA, Priya S, Pandian SK, Ravi AV. Inhibition of biofilm development of uropathogens by curcumin - An anti-quorum sensing agent from Curcuma longa. Food Chem 2014; 148:453-460.

28. Ozkaya $H, B a h a t ~ G$, Tufan A et al. Successful treatment of non-healing pressure ulcers with topical nacetyl cysteine. J Wound Care. 2015; 24(12):606-611.

29. Zhao T, Liu Y. N-acetylcysteine inhibit biofilms produced by Pseudomonas aeruginosa. BMC Microbiol 2010; 10(1):140.

30. Gupta SC, Patchva S, Koh W, Aggarwal BB. Discovery of curcumin, a component of golden spice, and its miraculous biological activities. Clin Exp Pharmacol Physiol. 2012; 39(3):283-299.

31. Scharstuhl A, Mutsaers HA, Pennings SW et al. Curcumin-induced fibroblast apoptosis and in vitro wound contraction are regulated by antioxidants and heme oxygenase: implications for scar formation. J Cell Mol Med. 2009; 13(4):712-725.

32. Jagetia GC, Rajanikant GK. Effect of curcumin on radiation-impaired healing of excisional wounds in mice. J Wound Care 2004; 13(3):107-109.

33. Deniz $M$, Borman $H$, Seyhan T, Haberal M. An effective antioxidant drug on prevention of the necrosis of zone of stasis: N-acetylcysteine. Burns 2013; 39(2):320-325.

34. Kheradpezhouh E, Panjehshahin MR, Miri R, Javidnia K, Noorafshan A, Monabati A et al. Curcumin protects rats against acetaminophen-induced hepatorenal damages and shows synergistic activity with N-acetyl cysteine. Eur J Pharmacol. 2010; 628(1-3):274-281.

35. Castro B, Bastida FD, Segovia T, López Casanova P, Soldevilla JJ, Verdú-Soriano J. The use of an antioxidant dressing on hard to- heal wounds: a multicentre, prospective case series. J Wound Care. 2017; 26 (12):742-75. 
36. Jiménez García JF, Abad García MM, Ortiz Villegas L, Zarco Marín JM, Expósito Torres R, García Fernández FP. Cicatrización de úlceras venosas complejas mediante el control del estrés oxidativo. Rev ROL Enferm. 2018; 41(11-12):739-744.

37. Restrepo-Medrano JC, Verdú Soriano J. Development of a wound healing index for chronic wounds. EWMA Journal. 2012; 12(2):39-46.

38. Lee M, Han SH, Choi WJ, Chung KH, Lee JW. Hyaluronic acid dressing (Healoderm) in the treatment of diabetic foot ulcer: A prospective, randomized, placebo-controlled, single-center study. Wound Repair Regen. 2016;24(3):581-8.

39. Kundin J.A new way to size up a wound. Am J Nurs. 1989; 89(2): 206-7.

40. Verdú Soriano J, López Casanova P, Rodríguez Palma M, García Fernández FP, Pancorbo Hidalgo PL, Soldevilla Ágreda JJ. HELCOS. Sistema integrado para el manejo de heridas. Rev ROL Enferm. 2018; 41(11-12): 778-783.

41. Integrated wound management system.2017. https://helcos.net/users/login . Accessed 25 Sep 2019.

42. Mao G, Goswami M, Kalen AL, Goswami PC, Sarsour EH. N-acetyl-L-cysteine increases MnSOD activity and enhances the recruitment of quiescent human fibroblasts to the proliferation cycle during wound healing. Molecular Biology Reports.2016;43(1):31-39.

43. Bell ML, Fiero M, Horton NJ, HsuCH. Handling missing data in RCTs; a review of the top medical journals. BMC Med. Res. Methodol. 2014; 14:118.

44. Sussman C, Bates-Jensen BM. Skin and Soft Tissue Anatomy and Wound Healing Physiology. In: Sussman C, Bates-Jensen BM. Wound Care. A collaborative Practice Manual for Health Professionals. China: Wolters Kluwer; 2012. p. 17-52.

\section{Additional Files}

Additional File 1. SPIRIT 2013 Checklist: Recommended items to address in a clinical trial protocol and related documents.

Additional File 2. Collaborating primary health care centres in the REOX study, and expected number of patients recruited.

\section{Figures}




\begin{tabular}{|c|c|c|c|c|c|}
\hline \multirow[b]{3}{*}{ Timepoints } & \multicolumn{5}{|c|}{ STUDY PERIOD } \\
\hline & \multirow{2}{*}{$\begin{array}{c}\text { ENROLLMENT } \\
\text { ALLOCATION } \\
\begin{array}{c}\text { Baseline } \\
\text { visit }\end{array}\end{array}$} & \multicolumn{4}{|c|}{ POST ALLOCATION } \\
\hline & & $\begin{array}{c}\text { 2-week } \\
\text { follow-up }\end{array}$ & $\begin{array}{c}\text { 4-week } \\
\text { follow-up }\end{array}$ & $\begin{array}{c}\text { 6-week } \\
\text { follow-up }\end{array}$ & $\begin{array}{c}\text { 8-week } \\
\text { follow-up/ } \\
\text { complete } \\
\text { healing }\end{array}$ \\
\hline \multicolumn{6}{|l|}{ ENROLLMENT } \\
\hline Informed consent & $x$ & & & & \\
\hline Eligibility screen & $x$ & & & & \\
\hline Inclusion/exclusion criteria & $x$ & & & & \\
\hline Allocation & $x$ & & & & \\
\hline \multicolumn{6}{|l|}{ INTERVENTIONS } \\
\hline \multicolumn{6}{|l|}{ Antioxidant dressing } \\
\hline \multicolumn{6}{|l|}{ Usual care dressing } \\
\hline \multicolumn{6}{|l|}{ ASSESMENTS } \\
\hline Sociodemographic characteristics & $x$ & & & & \\
\hline Clinical variables & $x$ & & & & \\
\hline Anthropometric variables & $x$ & & & & \\
\hline Medical drugs & $x$ & & & & \\
\hline Smoking habit & $x$ & & & & \\
\hline $\begin{array}{l}\text { Nutritional status } \\
\text { (MUST scale) }\end{array}$ & $x$ & & & & \\
\hline Pain level (VAS scale) & $x$ & & & & \\
\hline Doppler (if applicable) & $x$ & & & & \\
\hline $\begin{array}{l}\text { Treatment therapy compressive } \\
\text { (if applicable) }\end{array}$ & $x$ & & & & \\
\hline Previous wound management & $x$ & & & & \\
\hline Wound characteristics & $x$ & & & & \\
\hline $\begin{array}{l}\text { Wound Monitoring (RESVESCH } \\
2.0 \text { ) }\end{array}$ & & $x$ & $x$ & $x$ & $x$ \\
\hline Clinical photograph of wound & & $x$ & $x$ & $x$ & $x$ \\
\hline $\begin{array}{l}\text { Analysis of wound (HELCOS } \\
\text { Software) }\end{array}$ & & $x$ & $x$ & $x$ & $x$ \\
\hline Adverse events & & $x$ & $x$ & $x$ & $x$ \\
\hline
\end{tabular}

Figure 1 Standard Protocol Items: Recommendations for Interventional Trials (adapted from

SPIRIT figure) schedule of enrollment, interventions and assessments.

\section{Figure 1}

Standard Protocol Items: Recommendations for Interventional Trials (adapted from SPIRIT figure) schedule of enrollment, interventions and assessments. 


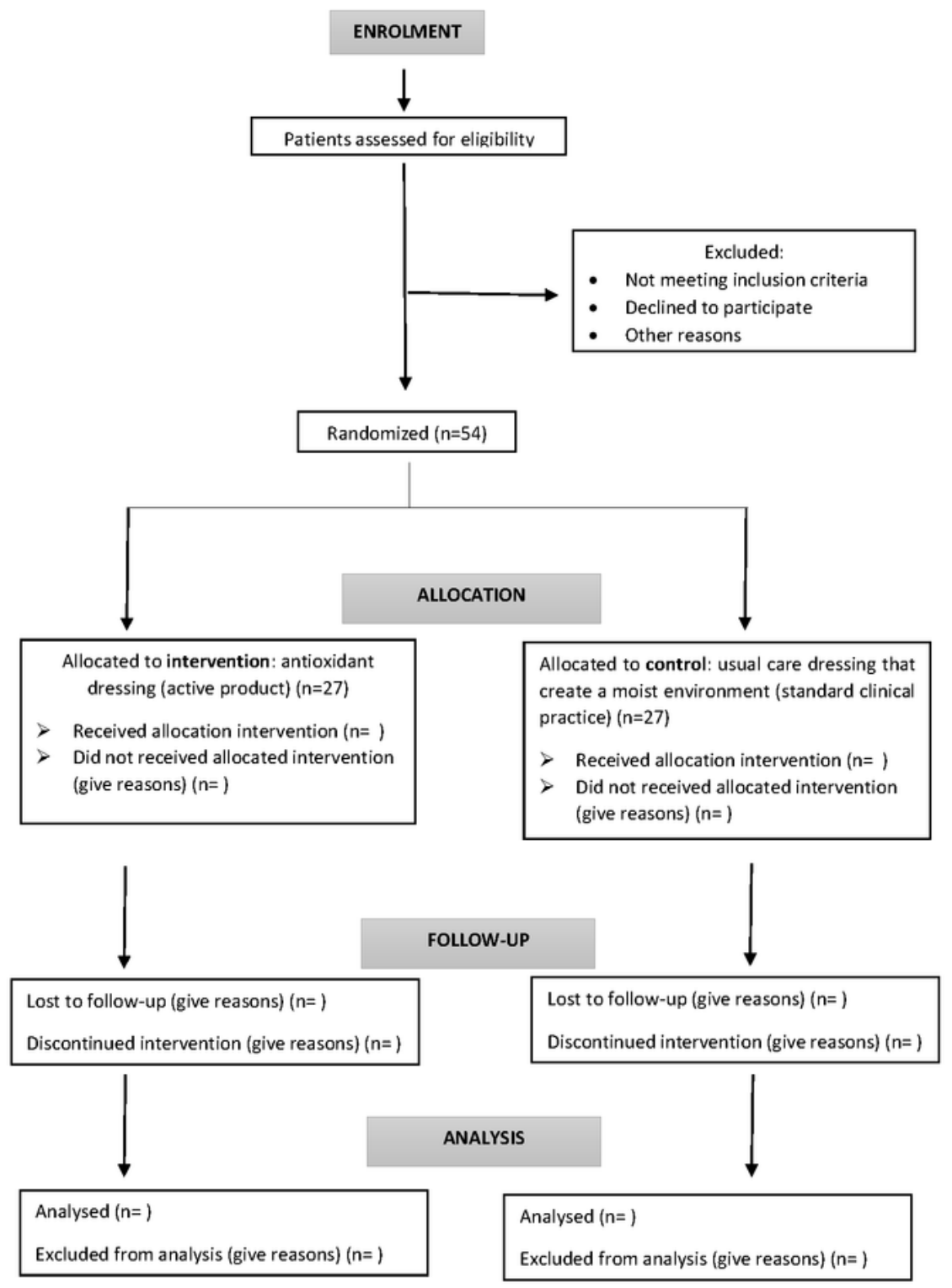

Figure 2. Schedule of enrolment.

Figure 2

Schedule of enrolment.

\section{Supplementary Files}

This is a list of supplementary files associated with this preprint. Click to download. 
- Additionalfile1SPIRIT.v2.pdf

- Additionalfile2Primaryhealthcarecentres.docx

- Table1.pdf 